\title{
ARTICLE
}

\section{The influence of glucose-lowering therapies on cancer risk in type 2 diabetes}

\author{
C. J. Currie • C. D. Poole • E. A. M. Gale
}

Received: 19 May 2009 / Accepted: 18 June 2009 / Published online: 2 July 2009

(C) Springer-Verlag 2009

\begin{abstract}
Aims/hypothesis The risk of developing a range of solid tumours is increased in type 2 diabetes, and may be influenced by glucose-lowering therapies. We examined the risk of development of solid tumours in relation to treatment with oral agents, human insulin and insulin analogues.

Methods This was a retrospective cohort study of people treated in UK general practices. Those included in the analysis developed diabetes $>40$ years of age, and started treatment with oral agents or insulin after 2000. A total of 62,809 patients were divided into four groups according to whether they received monotherapy with metformin or sulfonylurea, combined therapy (metformin plus sulfonylurea), or insulin. Insulin users were grouped according to treatment with insulin glargine, long-acting human insulin, biphasic analogue and human biphasic insulin. The outcome measures were progression to any solid tumour, or cancer of the breast, colon, pancreas or prostate. Confounding factors were accounted for using Cox proportional hazards models.

Results Metformin monotherapy carried the lowest risk of cancer. In comparison, the adjusted HR was 1.08 (95\% CI
\end{abstract}

\section{J. Currie $(\bowtie)$}

School of Medicine, Cardiff University,

The Pharma Research Centre, Cardiff MediCentre,

Cardiff CF14 4UJ, UK

e-mail: currie@cardiff.ac.uk

C. D. Poole

Department of Epidemiology, Pharmatelligence,

Cardiff, UK

E. A. M. Gale

Diabetes and Metabolism, School of Medicine, Bristol University, Bristol, UK
0.96-1.21) for metformin plus sulfonylurea, 1.36 (95\% CI 1.19-1.54) for sulfonylurea monotherapy, and $1.42(95 \%$ CI 1.27-1.60) for insulin-based regimens. Adding metformin to insulin reduced progression to cancer (HR 0.54, 95\% CI 0.43-0.66). The risk for those on basal human insulin alone vs insulin glargine alone was 1.24 (95\% CI 0.90-1.70). Compared with metformin, insulin therapy increased the risk of colorectal (HR 1.69, 95\% CI 1.23-2.33) or pancreatic cancer (HR 4.63, 95\% CI 2.64-8.10), but did not influence the risk of breast or prostate cancer. Sulfonylureas were associated with a similar pattern of risk as insulin.

Conclusions/interpretation Those on insulin or insulin secretagogues were more likely to develop solid cancers than those on metformin, and combination with metformin abolished most of this excess risk. Metformin use was associated with lower risk of cancer of the colon or pancreas, but did not affect the risk of breast or prostate cancer. Use of insulin analogues was not associated with increased cancer risk as compared with human insulin.

Keywords Cancer · Insulin · Insulin analogues · Metformin · Sulfonylureas $\cdot$ Survival $\cdot$ Type 2 diabetes

\section{Abbreviations \\ LVD Large vessel disease \\ OHA Oral hypoglycaemic agent \\ THIN The Health Information Network}

\section{Introduction}

Type 2 diabetes is associated with an increased risk of mortality from a range of solid tumours, including cancers of the colon, breast and pancreas [1]. Similar associations 
have been noted with central obesity and other conditions associated with increased levels of circulating insulin. These observations have given rise to the hypothesis that growth of these tumours, which are characterised by abnormal expression and function of the insulin-IGF-1 series of receptors $[2,3]$, is promoted by the trophic action of insulin interacting with these receptors. The cancer risk associated with diabetes may also be influenced by therapy: for example, the risk of colon cancer is higher in individuals on insulin [4], patients on metformin are less likely to be diagnosed with cancer [5], and the risk of mortality from solid tumours is lower for metformin than for exogenous insulin or sulfonylureas [6]. As recognition dawns that cancer should be numbered among the complications of diabetes, the possibility that therapies for diabetes may influence tumour progression is likely to attract increasing interest and concern. Furthermore, the observation that both endogenous insulin and exogenous insulin therapy are associated with tumour progression raises questions as to the safety of the insulin analogues, which have subtly modified receptor binding properties and accelerate the growth and proliferation of both healthy and tumour cell lines in culture $[7,8]$.

The present study examined the relative frequency with which cancer was diagnosed in patients receiving a range of therapies for type 2 diabetes, including human and analogue insulins. To establish the overall pattern of cancer risk using alternative glucose-lowering therapies, we compared four common treatment regimens for type 2 diabetes: metformin monotherapy, sulfonylurea monotherapy, combination therapy with metformin plus sulfonylureas, and all insulin-based therapies combined. We tested the hypotheses that therapies other than metformin would be associated with increased development of solid tumours in general, and of diabetes-associated cancers of the breast, colon and pancreas in particular. We also considered carcinoma of the prostate, which has a weak negative association with diabetes [9], and looked to see if combined treatment with metformin influenced these risks. Finally, we subdivided the insulin-based therapies to test the hypothesis that insulin glargine (A21Gly, B31Arg,B32Arg human insulin) or analogue mixes were associated with a greater risk of cancer than insulin of human origin.

\section{Methods}

Data source This was a retrospective cohort study of people treated in UK general practices participating in The Health Information Network (THIN). Created in 2002, THIN includes data from approximately 300 UK practices, and is similar in structure and scope to the General Practice
Research Database [10-14]. Patients included in THIN are similar in age, sex and geographic characteristics to the general UK population [10-12]. THIN includes records on 4.78 million patients, of which 2.26 million are currently active. Approximately $3 \%$ of patients are lost annually because of leaving a practice or death. The data are collected in a non-interventional way from the daily record keeping of physicians. The records are anonymised at the collection stage so that researchers have access to only encrypted identifiers for the physician's office and the patient. The database contains information on all past and current medical diagnoses, both acute and chronic (coded using Read codes), and prescribed medications (coded using British National Formulary codes). Unlike many other databases, THIN also includes laboratory values, which are electronically captured, and some aspects of physical examinations. Validation studies have been published and data collected in this way have previously been used to study diabetes $[10,11,15]$.

Patients were selected if they achieved cohort membership after 2000, shortly before the introduction of insulin glargine in the UK in mid-2000.

Study population Patients selected for analysis had a diagnosis of diabetes presenting later than 40 years of age, had received six or more sequential prescriptions for oral hypoglycaemic agents (OHAs), and other potential causes of secondary diabetes had not been recorded. Four primary cohorts were defined. Cohort 1 and Cohort 2 were defined by newly initiated OHA monotherapy (preceded by a wash-in period of $>6$ months, during which no OHAs were prescribed) with either metformin or a sulfonylurea, respectively. Cohort 3 was defined by a newly identified switch from OHA monotherapy with either metformin or sulfonylurea to an oral regimen involving both drugs in combination but no other concomitant class of OHA. Cohort 4 included those previously treated with OHAs who had been newly initiated on any insulin-based regimen. Cohort 4 was then subdivided when appropriate to assess the association between treatment subgroups and risk of cancer. The insulin subclasses were as follows: $4 \mathrm{a}$, insulin glargine with no other concomitant insulin; $4 \mathrm{~b}$, long-acting human insulin with no other concomitant insulin; 4c, biphasic insulin of human origin; and $4 \mathrm{~d}$, analogue biphasic insulin. In addition we wanted to provide some indication of the cancer risk of the various alternative diabetes therapies with regard to untreated diabetes. To this end, we included a preliminary analysis of the cancer risk in the aforementioned cohorts vs those with a diagnosis of type 2 diabetes who had no record of a diabetes-related medication, or those in other cohorts before prescription of their first diabetes-related medication. This comparison group thus represented a group of people with diet-treated 
diabetes, or who had yet to be given a diagnosis of type 2 diabetes, and who had no recorded exposure to diabetes medication.

Some patients with a sufficiently long treatment history had multiple cohort membership. To minimise selection bias, those patients who progressed to intensified therapy in more than one treatment class were included in the analysis for the previous cohort but had their membership censored at the date of treatment switching. Cohort membership was terminated by progression to a record of the primary or secondary outcomes of interest, right censoring at the final observation of the database, transfer out of the practice, or treatment switching. For all cohorts, the index date was fixed as the date of observed treatment initiation or switching to a treatment class of interest.

Patients with less than 6 months case history prior to the respective index date (as judged from the date of their first ever recorded prescription or laboratory test result) were excluded in order to improve the likelihood of observing the true start date for treatment intensification. According to figures from the THIN database, $95 \%$ of repeat prescriptions in general practice have a periodicity of 6 months or less. Patients with less than 6 months of exposure to an intensified regimen were excluded in order to ensure a sufficient degree of exposure in those remaining to potentially influence development of a solid tumour cancer.

Outcomes The primary outcome for this study was progression to the first record of any solid tumour cancer. The secondary outcome measure was progression to one of four specific solid tumour cancers recorded as the first cancer observed following treatment change, and only in people who had no record of a prior cancer: breast cancer, pancreatic cancer, colorectal cancer or prostate cancer. These cancers were selected because they were reported to be positively or negatively associated with diabetes [1, 9], and were

Table 1 Baseline characteristics for the four main treatment cohorts

\begin{tabular}{|c|c|c|c|c|c|}
\hline \multirow[t]{2}{*}{ Characteristic } & \multirow{2}{*}{$\begin{array}{l}\text { All } \\
\text { patients }\end{array}$} & \multicolumn{4}{|c|}{ Treatment cohort } \\
\hline & & $\begin{array}{l}\text { 1. Metformin } \\
\text { monotherapy }\end{array}$ & $\begin{array}{l}\text { 2. Sulfonylurea } \\
\text { monotherapy }\end{array}$ & $\begin{array}{l}\text { 3. Metformin+ } \\
\text { sulfonylurea }\end{array}$ & $\begin{array}{l}\text { 4. Insulin- } \\
\text { based }\end{array}$ \\
\hline Number of patients & 62,809 & 31,421 & 7,439 & 13,882 & 10,067 \\
\hline Age (years) & $62.0 \pm 14.6$ & $58.6 \pm 15.2$ & $70.0 \pm 13.9$ & $64.4 \pm 12.4$ & $63.7 \pm 12.9$ \\
\hline Sex, $\%$ female & 46.3 & 48.9 & 45.1 & 42.1 & 44.6 \\
\hline Smoking, \% ever smoked & 66.4 & 65.1 & 62.8 & 68.5 & 70.4 \\
\hline Diabetes duration (years) & $2.9(1.7)$ & $1.5(0.4)$ & $1.9(1.5)$ & $4.4(3.8)$ & $6.2(5.8)$ \\
\hline \multicolumn{6}{|l|}{ Weight $(\mathrm{kg})$} \\
\hline Men & $92.1 \pm 18.6$ & $95.9 \pm 18.8$ & $80.4 \pm 14.3$ & $90.9 \pm 17.5$ & $89.7 \pm 18.6$ \\
\hline Women & $82.1 \pm 19.3$ & $86.2 \pm 19.2$ & $68.0 \pm 15.0$ & $79.7 \pm 18.0$ & $79.8 \pm 18.7$ \\
\hline Systolic BP (mmHg) & $141 \pm 17$ & $142 \pm 17$ & $143 \pm 19$ & $141 \pm 16$ & $140 \pm 16$ \\
\hline Total cholesterol (mmol/l) & $5.0 \pm 1.2$ & $5.2 \pm 1.2$ & $5.1 \pm 1.3$ & $4.7 \pm 1.0$ & $4.7 \pm 1.1$ \\
\hline $\mathrm{HbA}_{1 \mathrm{c}}(\%)$ & $8.7 \pm 1.7$ & $8.4 \pm 1.8$ & $8.4 \pm 2.0$ & $8.6 \pm 1.4$ & $9.4 \pm 1.7$ \\
\hline \multicolumn{6}{|l|}{ Prior vascular disease $(\%)$} \\
\hline Large vessel disease & 22 & 18 & 26 & 23 & 30 \\
\hline Eye problems (some) & 9 & 5 & 6 & 11 & 20 \\
\hline Eye problems (SVL/blindness) & 2 & 1 & 3 & 2 & 3 \\
\hline No renal disease & 27 & 31 & 42 & 17 & 17 \\
\hline Mild renal disease & 44 & 46 & 28 & 51 & 43 \\
\hline Moderate renal disease & 25 & 21 & 26 & 30 & 32 \\
\hline End-stage renal disease & 4 & 3 & 4 & 3 & 8 \\
\hline \multicolumn{6}{|l|}{ General morbidity } \\
\hline Charlson index (adjusted) & $3.6(4)$ & $3.2(3)$ & $4.6(4)$ & $3.8(4)$ & $4.2(4)$ \\
\hline Charlson index (unadjusted) & $1.8(1)$ & $1.6(1)$ & $2.0(1)$ & $1.8(1)$ & $2.2(2)$ \\
\hline GP contacts in previous year & $7.7(6)$ & $6.7(5)$ & $6.1(4)$ & $8.3(7)$ & $10.8(9)$ \\
\hline Cancer morbidity: previous solid tumour cancers (\%) & 5.3 & 4.3 & 7.6 & 5.5 & 6.4 \\
\hline Total observation time (years) & 152,065 & 71,261 & 17,553 & 34,909 & 28,342 \\
\hline
\end{tabular}

Data are presented as mean (median), mean $\pm \mathrm{SD}$ or $\%$, unless specified otherwise

GP, general practitioner; SVL, severe visual loss 
examined as an individual secondary endpoint when the numbers of events allowed. Finally, with regard to breast cancer, a cancer that has given rise to concern with respect to insulin glargine, we undertook an analysis that compared those patients with any exposure to insulin glargine alone to exposure to any other insulin as a single group. This was needed to increase the statistical power of the comparison, which included relatively few breast cancer events.

Available baseline characteristics and/or covariates Baseline characteristics/covariates available for these real-life data from general practice included age, sex, systolic BP, total cholesterol, weight and weight change, BMI, smoking status, baseline general morbidity, prior large vessel disease (LVD), retinopathy, evidence of renal impairment (identified by a frank diagnosis of microalbuminuria or macroalbuminuria or end-stage renal disease [dialysis or transplant], estimated glomerular filtration rate of less than $45 \mathrm{ml} / \mathrm{min}$, or serum creatinine in excess of $130 \mu \mathrm{mol} / \mathrm{l}), \mathrm{HbA}_{1 \mathrm{c}}$ and records of prior solid tumour cancer. Concomitant metformin was introduced as a covariate into the Cox models for the insulin-regimen subgroup since it has been reported to be associated with a reduced cancer risk $[5,6]$.

Statistical methods The following four covariates were deliberately selected for inclusion in an initial Cox proportional hazards model before addition of the factor of interest: age, sex, smoking status and a diagnosis of a prior cancer. This model was tested and, as expected, all covariates were found to be highly significant. Other potential covariates were then additionally tested to determine their value in specifying the model, and these included, in various ways, $\mathrm{HbA}_{1 \mathrm{c}}$, diabetes duration and weight. None was found to be significant when added to the above model. Threshold statistical significance was set at

Table 2 Baseline characteristics for the insulin regimen subcohorts

\begin{tabular}{|c|c|c|c|c|c|}
\hline \multirow[t]{2}{*}{ Characteristic } & \multirow{2}{*}{$\begin{array}{l}\text { All } \\
\text { patients }\end{array}$} & \multicolumn{4}{|c|}{ Insulin treatment cohorts-starting regimen } \\
\hline & & $\begin{array}{l}\text { 4a. Insulin } \\
\text { glargine }\end{array}$ & $\begin{array}{l}\text { 4b. Human long- } \\
\text { acting insulin }\end{array}$ & $\begin{array}{l}\text { 4c. Human } \\
\text { biphasic insulin }\end{array}$ & $\begin{array}{l}\text { 4d. Analogue } \\
\text { biphasic insulin }\end{array}$ \\
\hline Number of patients & 8,034 & 2,286 & 1,262 & 2,003 & 2,483 \\
\hline Age (years) & $64.3 \pm 12.4$ & $65 \pm 12.6$ & $64.7 \pm 12.5$ & $66.3 \pm 12$ & $61.8 \pm 12.2$ \\
\hline Sex, $\%$ female & 44.2 & 45.5 & 47.0 & 45.4 & 40.5 \\
\hline Smoking, $\%$ ever smoked & 70.8 & 70.1 & 69.0 & 69.5 & 73.4 \\
\hline Diabetes duration (years) & $6.2(5.9)$ & $6.7(6.3)$ & $5.9(5.5)$ & $6.2(6.0)$ & $6.0(5.5)$ \\
\hline \multicolumn{6}{|l|}{ Weight $(\mathrm{kg})$} \\
\hline Men & $89.7 \pm 18.5$ & $90.9 \pm 18.7$ & $90.8 \pm 19.6$ & $86.5 \pm 17.1$ & $90.1 \pm 18.5$ \\
\hline Women & $79.5 \pm 18.2$ & $80.6 \pm 18.5$ & $78.8 \pm 17.4$ & $77.1 \pm 17.9$ & $80.4 \pm 18.2$ \\
\hline Systolic BP (mmHg) & $140 \pm 16$ & $140 \pm 16$ & $142 \pm 16$ & $142 \pm 18$ & $139 \pm 16.0$ \\
\hline Total cholesterol (mmol/l) & $4.7 \pm 1.2$ & $4.5 \pm 1.0$ & $4.8 \pm 1.2$ & $4.9 \pm 1.2$ & $4.7 \pm 1.2$ \\
\hline $\mathrm{HbA}_{1 \mathrm{c}}(\%)$ & $9.4 \pm 1.7$ & $9.4 \pm 1.6$ & $9.4 \pm 1.6$ & $9.4 \pm 1.8$ & $9.5 \pm 1.7$ \\
\hline \multicolumn{6}{|l|}{ Prior vascular disease $(\%)$} \\
\hline Large vessel disease & 31 & 27 & 27 & 37 & 32 \\
\hline Eye problems (some) & 20 & 22 & 19 & 20 & 21 \\
\hline Eye problems (SVL/blindness) & 3 & 2 & 3 & 4 & 2 \\
\hline No renal disease & 17 & 10 & 23 & 24 & 16 \\
\hline Mild renal disease & 43 & 46 & 44 & 32 & 44 \\
\hline Moderate renal disease & 33 & 35 & 28 & 35 & 31 \\
\hline End-stage renal disease & 8 & 9 & 6 & 6 & 8 \\
\hline \multicolumn{6}{|l|}{ General morbidity } \\
\hline Charlson index (adjusted) & $4.2(4)$ & $4.2(4)$ & $4.1(4)$ & $4.6(4)$ & $4.0(4)$ \\
\hline Charlson index (unadjusted) & $2.2(2)$ & $2.1(2)$ & $2.1(2)$ & $2.4(2)$ & $2.2(2)$ \\
\hline GP contacts in previous year & $10.7(9)$ & $10.9(9)$ & $9.8(8)$ & $10.6(9)$ & $11.1(9)$ \\
\hline Cancer morbidity: previous solid cancers $(\%)$ & 6.3 & 6.1 & 5.2 & 7.3 & 6.2 \\
\hline Total observation time (years) & 23,365 & 4,934 & 4,244 & 7,378 & 6,809 \\
\hline
\end{tabular}

Data are presented as mean (median), mean $\pm \mathrm{SD}$ or $\%$, unless specified otherwise

GP, general practitioner; SVL, severe visual loss 
the conventional level of $p=0.05$, and $95 \%$ CIs are given for HRs. With regard to progression to specific cancers, patients who had a previous cancer were excluded. The observational timescale for each individual was from cohort membership to a cancer event or death or switching to another cohort or their final recorded data (censorship). With regard to the additional cohort of patients who had not progressed to receipt of glucose-lowering medications, the index date for these patients was their first recorded date of any prescribed medication plus an observational run-in period of 3 years to allow for the identification of prior cancer.

\section{Results}

From an initial cohort of 170,000 patients (randomly selected by the data vendor from those meeting the initial selection criteria), some 85,200 individual patients met the criteria for at least one cohort. This was further reduced to 62,809 patients by selecting only those who achieved cohort membership after the year 2000 (Table 1). Membership of the individual cohorts was as follows: 31,421 (50\%) patients treated with metformin monotherapy; 7,439 (12\%) patients with sulfonylurea monotherapy; 13,882 (22\%) patients with combination therapy with metformin plus sulfonylureas, and 10,067 (16\%) patients treated with an insulin-based regimen. Among the insulin-treated patients, $44 \%$ were also represented in the combination therapy cohort. Within the combination therapy cohort, $23 \%$ were also represented in the monotherapy cohorts. Total follow-up time was 152,065 person-years; 17,553 person-years in the sulfonylurea group, the least numerous cohort (Table 1).

Baseline characteristics by the four general cohorts The common pattern of type 2 diabetes treatment progression and intensification in the UK was reflected in the baseline characteristics of the four respective treatment cohorts. People initiated on metformin monotherapy were, on average, youngest (59 years old) (Table 1). Those initiated on OHA combination therapy and insulin-based therapies, on average, were of similar age (64 years), whereas those initiated with sulfonylureas were older (70 years). The duration of diabetes varied in accordance with this pattern: the mean was 2.9 (median 1.7) years overall, and $1.5(0.4)$, 1.9 (1.5), 4.4 (3.8) and 6.2 (5.8) years for metformin monotherapy, sulfonylurea monotherapy, OHA combination therapy and insulin-based therapies, respectively. These observations illustrate that there were, understandably, considerable differences between the four general cohorts. A detailed comparison of the baseline characteristics of the four general treatment cohorts is listed in
Table 1. Features of note that may have affected this study included increased smoking incidence in insulin-treated patients; $70 \%$ had ever smoked as against $66 \%$ for the group as a whole. People who started sulfonylurea monotherapy were lighter; the average weight in this cohort was $80 \mathrm{~kg}$ for men and $68 \mathrm{~kg}$ for women, compared with 92 and $82 \mathrm{~kg}$, respectively, for the group as a whole. The crude prevalence of prior solid tumour cancers also differed by cohort at baseline, varying from $4.3 \%$ in patients treated with metformin monotherapy, to $7.6 \%$ those who received sulfonylurea monotherapy.
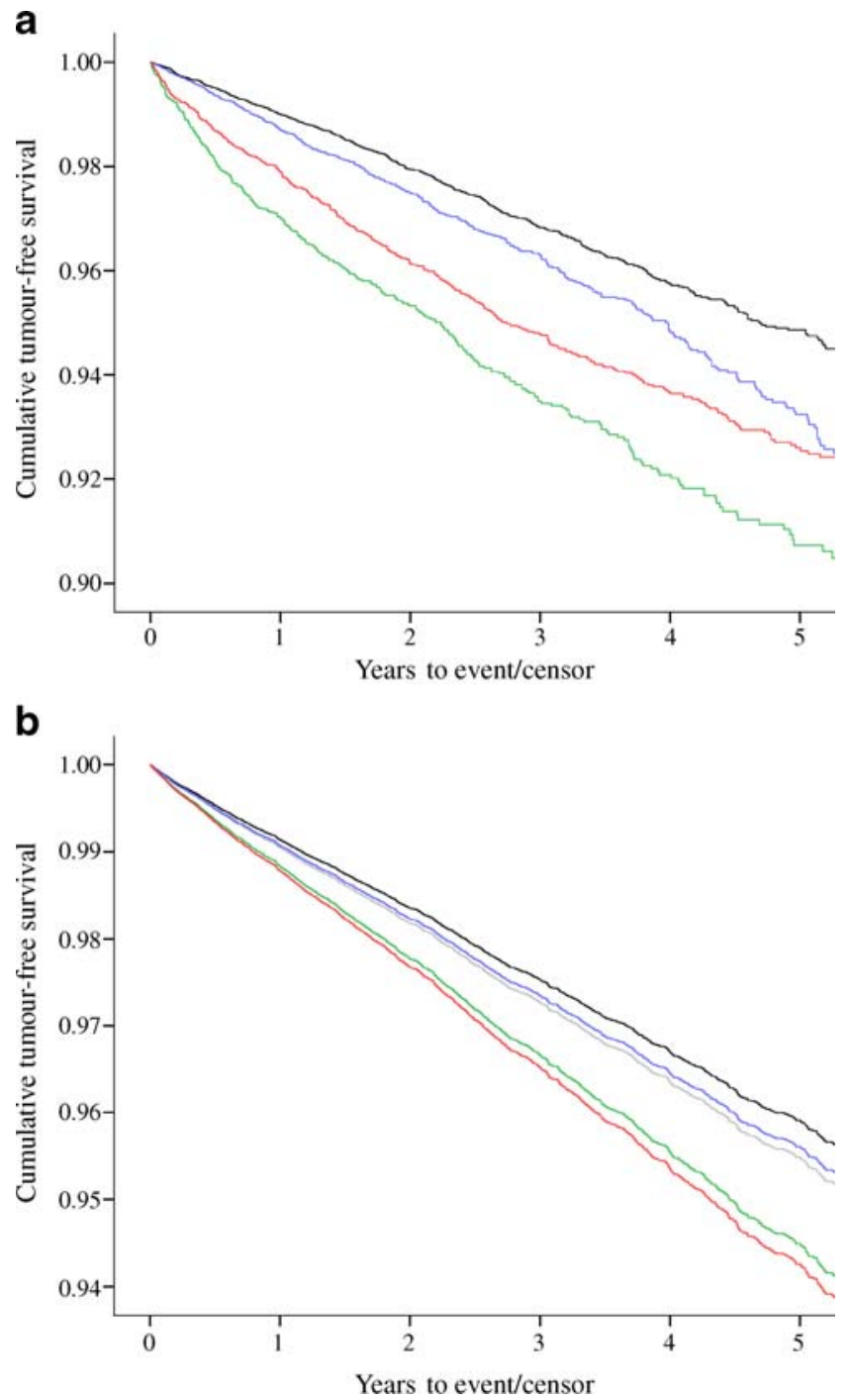

Fig. 1 Rate of progression of solid tumour cancers in people with diabetes receiving alternative glucose-lowering therapies (metformin monotherapy, black lines; sulfonylurea monotherapy, green lines; sulfonylurea plus metformin, blue lines; insulin-based therapy, red lines) and a group with no diabetes treatment exposure (grey lines). a Unadjusted (Kaplan-Meier curve). b Adjusted for confounding factors (age, sex, smoking status and prior cancer) using a Cox proportional hazards model 
Table 3 Cox proportional hazards model for progression to solid tumour cancers in people treated with metformin monotherapy, sulfonylurea monotherapy, combination therapy (metformin plus sulfonylureas) and insulin-based therapies (Cohorts 1 to 4 , respectively)

The model included 61,368 patients and 2,051 events

${ }^{\mathrm{a}} \mathrm{HRs}$ are relative to metformin monotherapy (Cohort 1)

\begin{tabular}{llllc}
\hline Covariate & HR & \multicolumn{2}{l}{$95 \%$ CI for HR } & \multirow{2}{*}{$p$ value } \\
& & Lower & Upper & \\
\hline Treatment $^{\mathrm{a}}$ & & & & \\
$\quad$ Sulfonylureas (Cohort 2) & 1.36 & 1.19 & 1.54 & $<0.001$ \\
$\quad$ Metformin plus sulfonylureas (Cohort 3) & 1.08 & 0.96 & 1.21 & 0.21 \\
$\quad$ Insulin-based therapies (Cohort 4) & 1.42 & 1.27 & 1.60 & $<0.001$ \\
Sex (female vs male) & 0.88 & 0.81 & 0.97 & 0.01 \\
Age at baseline (years) & 1.04 & 1.04 & 1.05 & $<0.001$ \\
Smoked (ever vs never) & 1.35 & 1.22 & 1.49 & $<0.001$ \\
Prior solid tumour cancer (yes vs no) & 3.86 & 3.46 & 4.31 & $<0.001$ \\
\hline
\end{tabular}

Baseline characteristics by insulin-treatment subcohorts The 8,034 people representing the four selected insulin subcohorts (Cohorts $4 \mathrm{a}$ to $4 \mathrm{~d}$ ), accounted for $80 \%$ of the 10,067 patients with newly initiated insulin-based regimens (Tables 1 and 2). The reasons for starting a particular insulin regimen in patients with type 2 diabetes are not clear.

Although less obvious than the differences observed between the four main cohorts above, there were systematic differences between those starting the four insulin regimens. For example, people initiated with analogue biphasic insulin were, on average, younger (62 years old) than people initiated with human biphasic insulin (66 years old) (Table 2; $p<$ 0.001). The average age of both Cohort $4 \mathrm{a}$ (insulin glargine alone) and Cohort 4b (human basal insulin alone) was 65 years. Thus, Cox proportional hazards models were necessary to adjust for these systematic differences.

Risk of progression to all solid tumour cancers Across all patients, 2,106 people progressed to a record of a first solid tumour cancer $(1.1 \%$ annual incidence). The crude inci- dence of solid tumour cancer in only those people who had no prior solid tumour cancer was $0.9 \%, 1.6 \%, 1.1 \%$ and $1.3 \%$ per year for Cohorts 1 to 4 , respectively. The unadjusted rate of progression to solid tumour cancer for the four general cohorts is illustrated in the Kaplan-Meier curve in Fig. 1. The pattern of unadjusted risk at a gross level appeared to reflect the systematic differences in the baseline characteristics, whereby the younger metformin monotherapy-treated patients had the lowest cancer incidence, and the older sulfonylurea monotherapy-treated patients had the highest. Following adjustment for confounding factors, the pattern of unadjusted risk altered to present an alternative, distinct pattern (Table 3, Fig. 1). Whilst metformin monotherapy still had the lowest risk, the insulin-based regimens then had the highest risk of progression to solid tumour cancer (HR 1.42, CI 1.271.60). The adjusted HR of a solid tumour cancer for those starting sulfonylurea monotherapy was 1.36 (95\% CI 1.19 1.54), and for OHA combination therapy was 1.08 (95\% CI 0.96-1.21). In the same Cox proportional hazards model

Table 4 Cox proportional hazards model for progression to all solid tumours in people treated with alternative insulin regimens

\begin{tabular}{|c|c|c|c|c|}
\hline \multirow[t]{2}{*}{ Covariate } & \multirow[t]{2}{*}{ HR } & \multicolumn{2}{|c|}{$95 \% \mathrm{CI}$ for $\mathrm{HR}$} & \multirow[t]{2}{*}{$p$ value } \\
\hline & & Lower & Upper & \\
\hline \multicolumn{5}{|l|}{ Initial insulin regimen $^{\mathrm{a}}$} \\
\hline Human basal insulin (Cohort 4b) & 1.24 & 0.90 & 1.70 & 0.19 \\
\hline Human biphasic insulin (Cohort 4c) & 0.88 & 0.66 & 1.19 & 0.42 \\
\hline Analogue biphasic insulin (Cohort 4d) & 1.02 & 0.76 & 1.37 & 0.91 \\
\hline Sex (female vs male) & 0.92 & 0.75 & 1.14 & 0.45 \\
\hline Age at insulin initiation (years) & 1.04 & 1.03 & 1.05 & $<0.001$ \\
\hline Smoking status (ever vs never) & 1.56 & 1.21 & 2.02 & 0.001 \\
\hline Concomitant metformin (yes vs no) & 0.54 & 0.43 & 0.66 & $<0.001$ \\
\hline Prior solid tumour (yes vs no) & 3.78 & 2.92 & 4.90 & $<0.001$ \\
\hline
\end{tabular}

The alternative insulin regimens were insulin glargine, human basal insulin, analogue biphasic insulin and human biphasic insulin (Cohorts 4 a to $4 \mathrm{~d}$, respectively)

The model included 7,897 patients and 373 solid tumour events

${ }^{\text {a }}$ HRs are relative to basal glargine (Cohort 4a) 


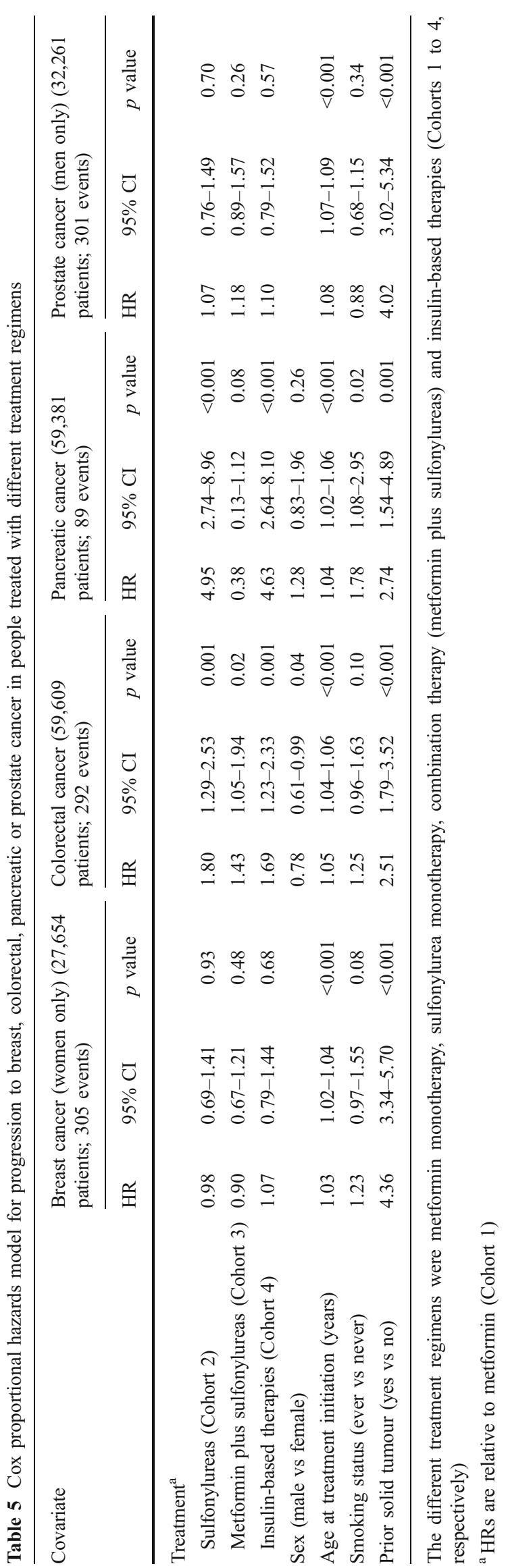



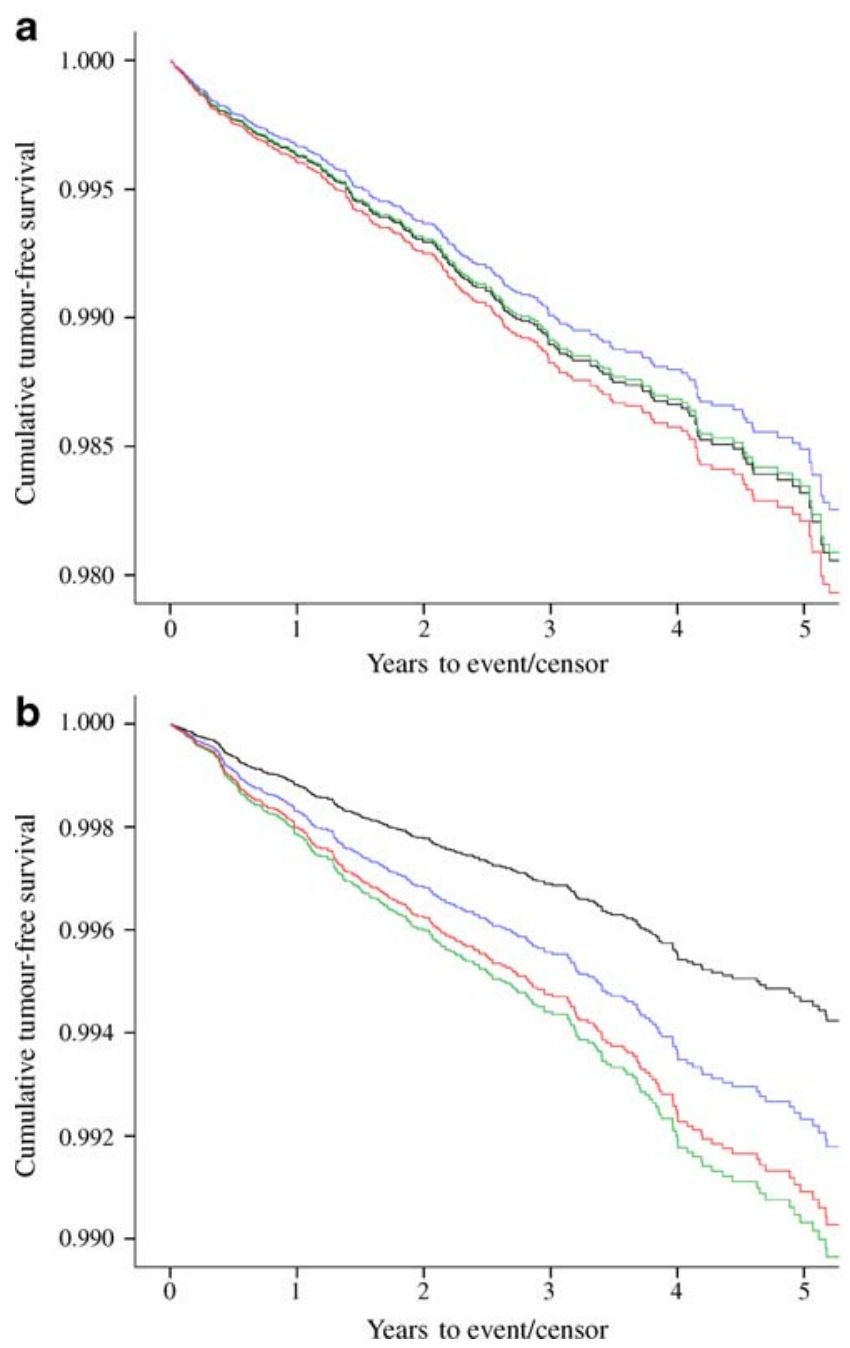

Fig. 2 Estimates of the survivor function showing progression to individual solid tumours (breast [a], colorectal $[\mathbf{b}]$, pancreatic $[\mathbf{c}]$ and prostate [d]) in people treated with metformin only (black lines), sulfonylurea only (green lines), metformin plus sulfonylurea (blue

(Table 3), the HR of solid tumour cancer in those who had smoked vs those who had never smoked was 1.35 (95\% CI $1.22-1.49$ ). The inclusion or exclusion of prior solid tumour cancers had little impact on these findings; for example, the HR for insulin-based regimens vs metformin monotherapy decreased slightly to 1.35 (95\% CI 1.19-1.54) following exclusion of patients with prior solid tumour cancers.

The specific insulin regimens did not differ with regard to progression to all solid tumour cancers (Table 4). It was possible to introduce the covariate 'concomitant metformin at any time during insulin exposure' into this model. Metformin use was associated with lower risk of cancer in these insulintreated patients (HR 0.54, 95\% CI 0.43-0.66; Table 4).

Risk of progression to breast, colorectal, pancreatic or prostate cancer The results for each of the four cancers
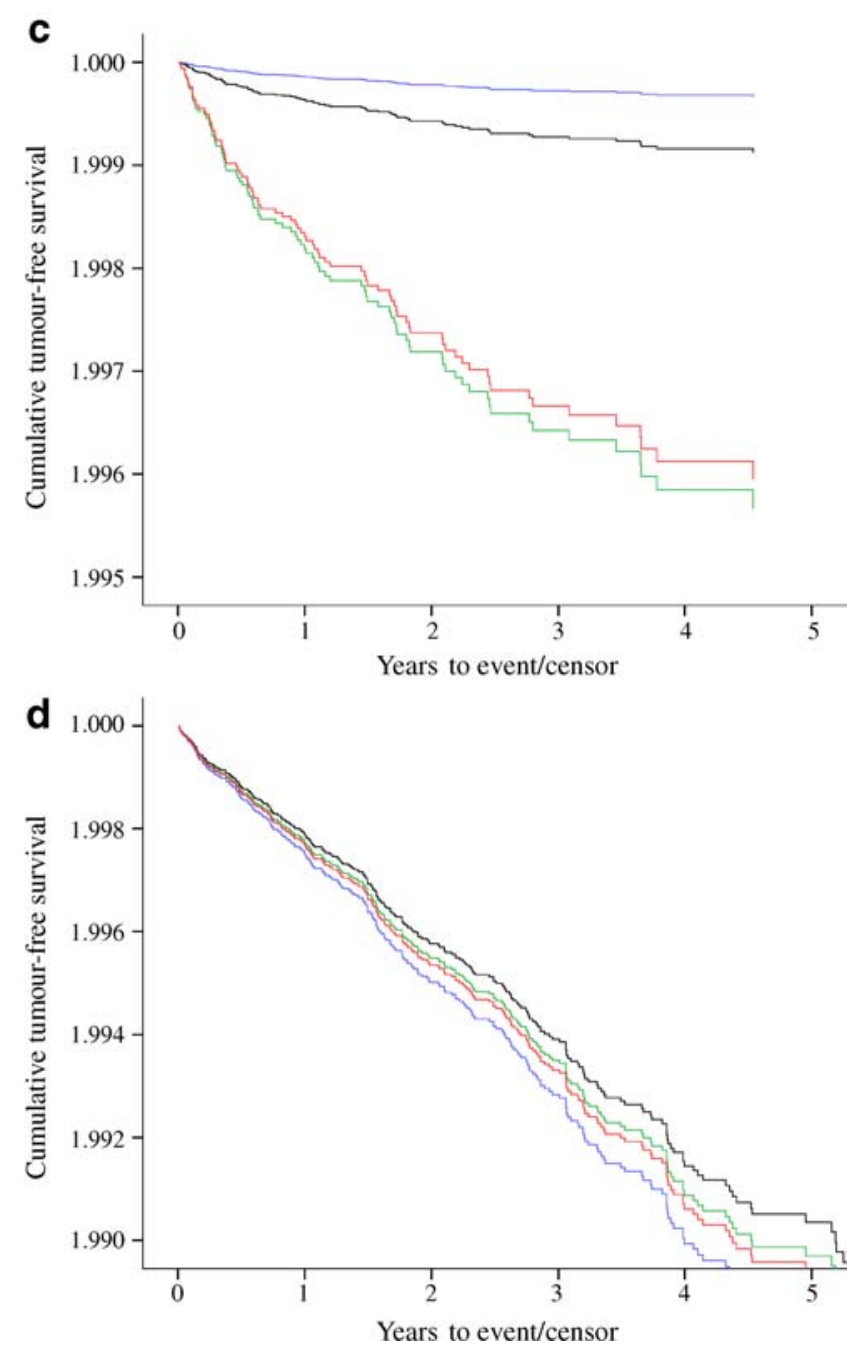

lines) or insulin-based therapy (red lines) (Cohorts 1 to 4 , respectively), following adjustment for confounding factors using Cox proportional hazards models (Table 5)

considered individually were largely similar to those for all solid tumour cancers in terms of the order of the cohorts by risk (Table 5). With regard to the combined risk of progression to breast or colorectal or prostate cancer, compared with metformin monotherapy, the HRs for sulfonylurea monotherapy, oral combination therapy and insulin-based therapies were 1.62 (95\% CI 1.30-2.01), 1.07 (95\% CI $0.87-1.31)$ and 1.55 (95\% CI $1.27-1.89)$, respectively. No difference emerged for breast cancer alone or prostate cancer alone between the four main therapy classes (Table 5, Fig. 2), but large differences were seen for colorectal cancer alone and pancreatic cancer alone. The HR for insulin treatment relative to metformin monotherapy was 1.69 (95\% CI 1.23-2.33) for colorectal cancer, and 4.63 (95\% CI 2.64-8.10; Table 5) for pancreatic cancer. 
Table 6 Cox proportional hazards model for progression to breast, colorectal or pancreatic cancer in people treated with alternative insulin regimens

\begin{tabular}{|c|c|c|c|c|}
\hline \multirow[t]{2}{*}{ Covariate } & \multirow[t]{2}{*}{ HR } & \multicolumn{2}{|c|}{$95 \% \mathrm{CI}$ for $\mathrm{HR}$} & \multirow[t]{2}{*}{$p$ value } \\
\hline & & Lower & Upper & \\
\hline \multicolumn{5}{|l|}{ Initial regimen $^{\mathrm{a}}$} \\
\hline Human long-acting insulin (Cohort 4b) & 1.17 & 0.70 & 1.94 & 0.55 \\
\hline Human biphasic insulin (Cohort 4c) & 0.76 & 0.47 & 1.24 & 0.27 \\
\hline Analogue biphasic insulin (Cohort 4d) & 1.01 & 0.63 & 1.63 & 0.95 \\
\hline Sex (male vs female) & 2.23 & 1.55 & 3.22 & $<0.001$ \\
\hline Age at insulin initiation (years) & 1.05 & 1.03 & 1.07 & $<0.001$ \\
\hline Smoking status (ever vs never) & 1.49 & 1.01 & 2.20 & 0.05 \\
\hline Concomitant metformin (yes vs no) & 0.73 & 0.51 & 1.04 & 0.08 \\
\hline Prior solid tumour (yes vs no) & 3.70 & 2.41 & 5.67 & $<0.001$ \\
\hline
\end{tabular}

The alternative insulin regimens were insulin glargine, neutral protamine Hagedorn (NPH) insulin, analogue biphasic insulin and human biphasic insulin (Cohorts 4 a to $4 \mathrm{~d}$, respectively)

The model included 7,659 patients and 135 solid tumour events

${ }^{a}$ HRs are relative to insulin glargine (Cohort $\left.4 a\right)$

The subgroup of insulin-based therapies was too small for individual consideration of each cancer. We therefore examined a combined endpoint of breast, colorectal and pancreatic cancer; no differences were seen between therapies (Table 6). Combined metformin and insulin therapy showed a trend to reduced risk of this combined endpoint (HR 0.73, 95\% CI 0.51-1.04; Table 6). Analysis of glargine vs all other insulins as a single comparator group revealed no difference with respect to progression to breast cancer (HR 0.86, 95\% CI 0.42-1.75; Table 7). The number of events in this comparison was ten in the glargine group vs 38 in the remaining insulin group.

Risk of cancer vs patients with no exposure to diabetes medication It was possible to identify 14,304 patients who met the inclusion criteria, with 39,683 person-years of follow-up. Their mean (SD) age at baseline was 56.6 (17.3) years; $46 \%$ were women, and $3.7 \%$ had prior cancer. Using the baseline Cox model, the adjusted risk of progression to a solid tumour cancer in this untreated cohort was the same as that for metformin (HR 0.90, 95\% CI 0.79-1.03; Table 8, Fig. 1).

Table 7 Cox proportional hazards model for progression to breast cancer in people treated with glargine vs all other insulin regimens

The model included 3,273 patients and 48 breast cancer events (women only)

\section{Discussion}

Type 2 diabetes, central obesity and other conditions associated with insulin resistance are all associated with an increased risk of certain types of cancer. Diabetes carries an increased risk of breast [16], colon [4] and pancreatic [17] cancer, each of which features among the top five causes of cancer mortality in the USA [2]. Prostate cancer also features among the five leading causes of cancer death [2], but has a weak negative association with diabetes [9]. Growth of all these cancers is influenced by the insulinIGF-1 signalling axis, as suggested by observational studies showing an association between cancer risk and levels of circulating insulin [2]. Laboratory studies have demonstrated that insulin levels (often, but not always, supraphysiological) may have direct effects in vitro on growth, proliferation and resistance to apoptosis of cancer cells [7, 8]. It remains an open question as to whether, or to what extent, differences in circulating levels of insulin influence cancer progression in patients receiving treatment for diabetes, but answers to this question are urgently needed. The use of exogenous insulin is also associated

\begin{tabular}{lllll}
\hline Covariate & HR & \multicolumn{2}{l}{$95 \%$ CI for HR } & \multirow{2}{*}{$p$ value } \\
\cline { 3 - 4 } & & Lower & Upper & \\
\hline Glargine vs all other insulins & 0.86 & 0.42 & 1.75 & 0.67 \\
Age at insulin initiation (years) & 1.03 & 1.01 & 1.06 & 0.02 \\
Smoking status (ever vs never) & 1.21 & 0.67 & 2.17 & 0.53 \\
Concomitant metformin (yes vs no) & 0.88 & 0.48 & 1.63 & 0.69 \\
Prior solid tumour (yes vs no) & 4.22 & 2.14 & 8.32 & $<0.001$ \\
\hline
\end{tabular}


Table 8 Cox proportional hazards model for progression to all solid tumour cancers in those with no prior exposure to a diabetes-related medication vs Cohorts 1 to 4

The model included 75,672 patients and 2,434 solid tumour events

\begin{tabular}{lllll}
\hline Covariate & HR & \multicolumn{2}{l}{$95 \%$ CI for HR } & \multirow{2}{*}{$p$ value } \\
\cline { 3 - 4 } & & Lower & Upper & \\
\hline No diabetes medications (Cohort 0) vs & & & & \\
$\quad$ Metformin (Cohort 1) & 0.90 & 0.79 & 1.03 & 0.12 \\
Sulfonylureas (Cohort 2) & 1.23 & 1.06 & 1.42 & 0.01 \\
Metformin plus sulfonylureas (Cohort 3) & 0.97 & 0.85 & 1.12 & 0.69 \\
Insulin-based therapies (Cohort 4) & 1.28 & 1.11 & 1.47 & 0.001 \\
Sex (female vs male) & 0.86 & 0.79 & 0.93 & $<0.001$ \\
Age at insulin initiation (years) & 1.04 & 1.04 & 1.05 & $<0.001$ \\
Smoking status (ever vs never) & 1.27 & 1.16 & 1.39 & $<0.001$ \\
Prior solid tumour (yes vs no) & 3.95 & 3.57 & 4.38 & $<0.001$ \\
\hline
\end{tabular}

with an increase in all-cause mortality in type 2 diabetes $[18,19]$.

The association between diabetes and cancer has hitherto attracted relatively little attention from diabetes specialists, mainly because it was considered to be unavoidable. This situation changed when metformin was found to be associated with a reduced risk of cancer in an observational study of patients with diabetes [5], an effect that appears to be due to a direct action of metformin on the AMPactivated protein kinase (AMPK) signalling pathway, rather than being secondary to its clinical effects on insulin sensitivity and hyperinsulinaemia [20]. The effect of therapeutic manipulation of endogenous insulin levels on cancer risk is unknown. Furthermore, little is known as to the influence of exogenous insulin therapy on cancer progression, although insulin treatment is reportedly associated with a considerably increased risk of colon cancer: about a $20 \%$ increase in risk per year of therapy [4]. In both the sulfonylurea monotherapy and insulin groups, the risk of progression to colorectal cancer was increased by 70 $80 \%$, while the increase in risk of progression to pancreatic cancer approached 400\% (Table 5).

Our study confirms a previous report that showed a hierarchy of cancer risk according to treatment of type 2 diabetes [6], ranging from a nadir with metformin monotherapy through combination therapy with sulfonylurea, sulfonylurea alone, and insulin (Fig. 1). The observation that treatment with insulin secretagogues confers an increase in risk similar to that of insulin would seem to exclude an adverse property of the insulin formulation itself. The association between metformin and individual types of tumour has not hitherto been examined. Our observed lack of effect of metformin treatment on the risk of breast cancer was unexpected in the light of the in vitro observations [20] that provided the rationale for an intervention trial with metformin following surgery for breast cancer [21]. Prostate cancer was also unaffected, but the striking differential between the risk of metformin and other therapies with respect to carcinoma of the pancreas, a largely incurable condition [22], deserves further investigation. Other tumours, not examined here, may also prove to be responsive to metformin, and the marked protective effects implied by these observations suggest that metformin may have anti-cancer effects sufficient to justify its use outside the context of diabetes.

Finally, we went on to examine a possible difference in cancer risk between human and analogue insulins based on differences in receptor binding, and the in vitro observation of increased mitogenicity, as seen with insulin glargine in particular [7, 8]. No difference in overall cancer progression between human insulin and the insulin analogues was observed in this study, and we were unable to confirm an increased risk of breast cancer with insulin glargine compared with all other insulin therapies. The number of tumour events was, however, relatively low. There was, nevertheless, a striking reduction in risk association (HR 0.54, 95\% CI 0.43-0.66) when metformin was co-administered with insulin (Table 4). Important limitations to this analysis were that numbers were insufficient to perform separate analyses for diabetesrelated tumours, or for insulin detemir. Furthermore, the influence of insulin dose or duration of treatment could not be examined. A clear dose-response association would have added weight to these findings, and would allow the hypothesis of causality to be addressed more directly.

This analysis is subject to many of the limitations inherent to all observational studies, not least a multiplicity of potential confounders. These were minimised by restricting the analysis only to patients who had recently initiated treatment. Although those starting oral monotherapy were previously treatment-naive, those starting on oral combinations or insulin regimens may have already been exposed to prior glucose-lowering therapy, and-as might be expected-had a longer duration of diabetes (Table 1). The sulfonylurea monotherapy group was older than the metformin monotherapy group $(70.0 \pm 13.9$ years vs $58.6 \pm$ 
15.2 years), and weighed less than the other three groups. Glucose control at baseline was worse in those on insulin, with an $\mathrm{HbA}_{1 \mathrm{c}}$ of $9.4 \%$ (Table 1), and this was a potential confounder since the risk of colon cancer is also related to glucose control [23].

There may also have been some under-reporting of cancer by the general practitioners, and since we examined only the first cancer diagnosis for each endpoint, it is plausible that diagnoses may have been updated and changed. Furthermore, all observations are intermittent and rely on routine recording. There was, on the other hand, no reason to believe that these limitations would have had a differential impact on the groups included in this analysis; furthermore, any 'noise' within the data would be expected to disguise patterns of association rather than to create spurious ones. Finally, the use of Cox models was intended to eliminate some of the systematic bias when comparing the various cohorts, but a plausible, yet small, possibility remained that a systematic bias could account for these findings. This having been said, the analysis was based on a large number of individuals followed over several years, and the patterns elucidated are clear-cut, suggesting that these findings are reliable.

In conclusion, this analysis has confirmed that metformin therapy is associated with a similar risk of cancer development to that seen in diet-treated or undiagnosed individuals, but a reduced risk compared with that associated with sulfonylureas or insulin; this protective effect is also seen when metformin is combined with a sulfonylurea or insulin. Metformin therapy was associated with a greatly reduced risk of colorectal or pancreatic cancer, but no effect was seen in relation to cancer of the breast or prostate. Metformin appears to confer major nonglycaemic benefits with regard to cancer progression, as for cardiovascular disease [24], further strengthening its status as the treatment of first choice for type 2 diabetes. Insulin glargine and analogue premixes were not associated with a greater risk of cancer progression, but larger and more detailed analyses will be needed to establish their safety with greater confidence. Cancer should be recognised as an important complication of type 2 diabetes, and its risk can potentially be modified by lifestyle changes [2] and metformin. The effect of other diabetes therapies on cancer progression, if any, remain to be established. Finally, there is currently no evidence that insulin or sulfonylureas have a harmful effect on cancer development; we can only be sure that they were associated with a higher risk than metformin, which has known anti-cancer properties. Insulin therapy may be associated with a higher risk of cancer, as it is with cardiovascular disease [25], but it would be premature to assume a causal relationship. Even if such a relationship were to be established, it would need to be weighed against the life-giving benefits of insulin.
Acknowledgements This study received no funding from any source.

Duality of interest C. J. Currie and C. D. Poole have previously received funding from various manufacturers of diabetes-related products, including Eli Lilly, GlaxoSmithKline, Medtronic, Novo Nordisk, Roche and Sanofi-Aventis. E. A. M. Gale has no financial interests to declare.

\section{References}

1. Coughlin SS et al (2004) Diabetes mellitus as a predictor of cancer mortality in a large cohort of US adults. Am J Epidemiol 159:1160-1167

2. Giovannucci E, Michaud D (2007) The role of obesity and related metabolic disturbances in cancers of the colon, prostate and pancreas. Gastroenterology 132:2208-2225

3. Samami AA, Yakar S, LeRoith D, Brodt D (2007) The role of the IGF system in cancer growth and metastasis: overview and recent insights. Endocr Rev 28:20-47

4. Yang Y-X, Hennessy S, Lewis JD (2004) Insulin therapy and colorectal cancer risk among type 2 diabetes mellitus patients. Gastroenterology 127:1044-1050

5. Evans JMM, Donnelly LA, Emslie-Smith AM, Alessi DR, Morris AD (2005) Metformin and reduced risk of cancer in diabetic patients. BMJ 330:1304-1305

6. Bowker SL, Majumdar SR, Veugelers P, Johnson JA (2006) Increased cancer-related mortality for patients with type 2 diabetes who use sulfonylureas or insulin. Diabetes Care 29:254-258

7. Kurtzhals P, Schäffer L, Sørensen A et al (2000) Correlations of receptor binding and metabolic and mitogenic potencies of insulin analogs designed for clinical use. Diabetes 49:999-1005

8. Shukla A, Grisouard J, Ehemann V, Hermani A, Enzmann H, Mayer D (2009) Analysis of signaling pathways related to cell proliferation stimulated by insulin analogs in human mammary epithelial cell lines. Endocr Relat Cancer 16:429-441

9. Bonovas S, Filioussi K, Tsantes A (2004) Diabetes mellitus and risk of prostate cancer: a meta-analysis. Diabetologia 47:1071-1078

10. Gelfand JM, Margolis DJ, Dattani H (2005) The UK general practice research database. In: Strom BL (ed) Pharmacoepidemiology, 3rd edn. Wiley, Chichester, pp 337-346

11. Jick H, Jick SS (1991) Validation of information recorded on general practitioner based computerized data resource in the United Kingdom. BMJ 302:766-768

12. Lewis JD, Shinnar R, Bilker W, Wang X, Strom BL (2007) Validation studies of the health improvement network for pharmacoepidemiology studies. Pharmacoepidemiol Drug Saf 16:393-401

13. http://www.gprd.com/home/, accessed 17 September 2007

14. Office for National Statistics (1996) The general practice research database: information for researchers. Office for National Statistics, London, pp 1-26

15. Mulnier HE, Seaman HE, Raleigh VS, Soedamah-Muthu SS, Colhoun HM, Lawrenson RA (2006) Mortality in people with type 2 diabetes in the UK. Diabetic Med 23:516-521

16. Larsson SC, Mantzoros CS, Wolk A (2007) Diabetes mellitus and risk of breast cancer: a meta-analysis. Int J Cancer 121:856 862

17. Larsson SC, Orsini N, Wolk A (2005) Diabetes mellitus and risk of colorectal cancer: a meta-analysis. J Natl Cancer Inst 97:16791687

18. Eurich DT, McAlister FA, Blackburn DF et al (2007) Benefits and harms of antidiabetic agents in patients with diabetes and heart failure: systematic review. BMJ 335:497 
19. Margolis DJ, Hoffstad O, Strom BL (2008) Association between serious ischemic cardiac outcomes and medications used to treat diabetes. Pharmacoepidemiol Drug Saf 17:753-759

20. Zakikhani M, Dowling R, Fantus IG, Sonenberg N, Pollak M (2006) Metformin is an AMP kinase-dependent growth inhibitor for breast cancer cells. Cancer Res 66:10269-10273

21. Cazaniga M, Bonanni B, Guerrieri-Gonzaga A, Decensi A (2009) Is it time to test metformin in breast cancer trials? Cancer Epidemiol Biomarkers Prev 18:701-705

22. Hezel AF, Kimmelman AC, Stanger BZ, Bardeesy N, DePinho RA (2006) Genetics and biology of pancreatic cancer. Genes Dev 20:1218-1247
23. Khaw K-T, Wareham N, Bingham S, Luben R, Welch A (2004) Preliminary communication: glycated hemoglobin, diabetes, and incidental colorectal cancer in men and women: a prospective analysis from the European Prospective Investigation into Cancer-Norfolk Study. Cancer Epidemiol Biomarkers Prev 13:915-919

24. Holman RR, Paul SK, Bethel A, Matthews DR, Neil AW (2008) 10-year follow-up of intensive glucose control in type 2 diabetes (UKPDS 80). N Engl J Med 359:1577-1589

25. Spencer EA, Pirie KL, Stevens RJ et al (2008) Diabetes and modifiable risk factors for cardiovascular disease: the prospective Million Women Study. Eur J Epidemiol 23:793-799 